
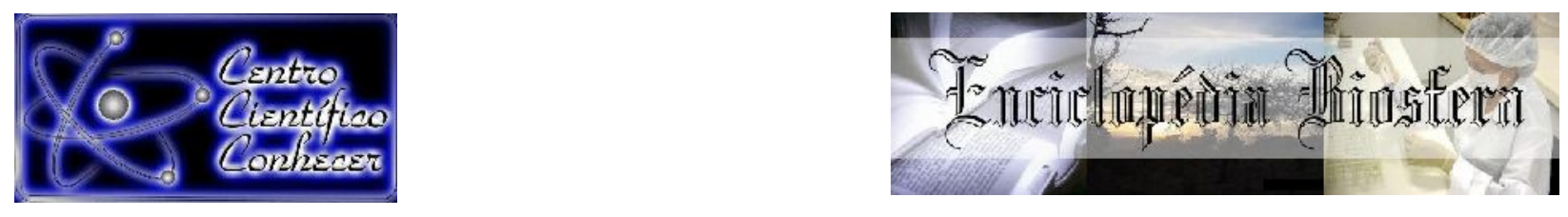

\title{
CONCENTRAÇÃO DE OZÔNIO TROPOSFÉRICO EM UBERLÂNDIA (MG) PELO MÉTODO DE SCHÖNBEIN
}

\author{
Arthur Dias Freitas ${ }^{1}$, Thaliene Alves da Silva ${ }^{1}$, Michael Douglas de Jesus Oliveira ${ }^{1}$, Yasmin \\ Pires Gonçalves ${ }^{1}$, Tatiane Pereira Santos Morais ${ }^{2}$ \\ ${ }^{1}$ Graduandos em Engenharia Ambiental pela Universidade Federal de Uberlândia \\ (arthurdiasfreitas@gmail.com) \\ ${ }^{2}$ Docente do Instituto de Ciências Agrárias, Universidade Federal de Uberlândia
}

Recebido em: 06/04/2019 - Aprovado em: 10/06/2019 - Publicado em: 30/06/2019

DOI: 10.18677/EnciBio_2019A38

\begin{abstract}
RESUMO
ozônio presente na baixa atmosfera se caracteriza como um poluente do ar por causar danos à saúde humana e ao meio ambiente, impactando principalmente o sistema respiratório e a vegetação natural e agrícola. Sua concentração é mais elevada no meio urbano devido aos seus precursores (NOx, $\mathrm{CO}$ e COVs) serem emitidos, majoritariamente, por indústrias e veículos automotores. O objetivo deste trabalho experimental foi determinar as concentrações de ozônio troposférico em cinco pontos de Uberlândia, o segundo município mais populoso do estado de Minas Gerais, pelo método dos amostradores passivos, proposto por Christian Friedrich Schönbein, o químico que identificou e nomeou o ozônio. Após oito horas de exposição, as concentrações de três dos pontos amostrados superaram o padrão estabelecido pela legislação. Os resultados obtidos permitem estimar a concentração de ozônio média em Uberlândia e sua distribuição espacial.
\end{abstract}

PALAVRAS-CHAVE: meio urbano, poluição do ar, química atmosférica.

\section{CONCENTRATION OF TROPOSPHERIC OZONE IN UBERLÂNDIA (MG) BY SCHÖNBEIN'S METHOD}

\begin{abstract}
The ozone present in the lower atmosphere is characterized as an air pollutant by causing damage to human health and the environment, impacting mainly the respiratory system and natural and agricultural vegetation. Its concentration is higher in the urban environment because of its precursors (NOx, CO and VOCs) are emitted, predominantly, by industries and automotive vehicles. The objective of this experimental work was to determine tropospheric ozone concentrations in five points of Uberlândia, the second most populous city in the state of Minas Gerais, by the method of passive samplers, proposed by Christian Friedrich Schönbein, the chemist who identified and named the ozone. After eight hours of exposure, the concentrations of three of the points sampled exceeded the standard established by the legislation. The results obtained allow us to estimate the average ozone concentration in Uberlândia and its spatial distribution.
\end{abstract}

KEYWORDS: air pollution, atmospheric chemistry, urban environment. 


\section{INTRODUÇÃO}

A atmosfera terrestre é dividida em cinco camadas de acordo com o comportamento da temperatura em diferentes altitudes. As duas camadas mais próximas da superfície do planeta contêm praticamente todo o ozônio $\left(\mathrm{O}_{3}\right)$ da atmosfera: cerca de $10 \%$ deste gás se encontra na troposfera (de 0 a $10 \mathrm{Km}$ sobre a superfície) e aproximadamente $90 \%$ está na estratosfera (de 10 a $50 \mathrm{Km}$ sobre a superfície). A parcela presente na estratosfera é comumente chamada de "ozônio bom", enquanto a presente na troposfera é denominada de "ozônio ruim" (SEINFELD; PANDIS, 2016).

O ozônio estratosférico, ou "bom", possui um papel fundamental na manutenção da vida na Terra, absorvendo a maior parte da radiação ultravioleta proveniente do Sol (BAIS et al., 2015). Porém, na baixa atmosfera, este mesmo gás é considerado um poluente por seus efeitos adversos ao sistema respiratório humano e à produtividade agrícola (MONKS et al., 2015). A concentração do ozônio estratosférico chega a $10.000 \mathrm{ppb}$ (partes por bilhão), enquanto a concentração do ozônio troposférico varia entre 20 e 100 ppb (HEGGLIN et al., 2015; SEINFELD; PANDIS, 2016). A resolução no 491 de 2018 do CONAMA (Conselho Nacional do Meio Ambiente) segue a orientação da OMS (Organização Mundial da Saúde) de 2005 e estabelece que o padrão para o ozônio na troposfera deve ser de $100 \mathrm{~g} / \mathrm{m}^{3}$ (ou 50 ppb) (BRASIL, 2018). As concentrações mais elevadas do ozônio "ruim" são encontradas nos centros urbanos, em decorrência da reação dos poluentes emitidos por indústrias e veículos automotores - poluentes primários - com a radiação solar, formando o ozônio, um poluente secundário. Tais poluentes precursores do ozônio são, principalmente, $\mathrm{NO}_{x}, \mathrm{CO}$ e COVs (MONKS et al., 2015).

Os riscos que podem oferecer as altas concentrações de ozônio, principalmente em ambientes urbanos, evidenciam a necessidade de monitoramento deste gás. Atualmente, diversas tecnologias estão disponíveis para este monitoramento, contemplando sensores em aviões, balões e satélites; aparelhos digitais; amostradores passivos; entre outros (HEGGLIN et al., 2015). O primeiro método de monitoramento de ozônio foi proposto pelo químico Christian Friedrich Schönbein, também responsável pela identificação e nomeação do ozônio. Seu método era simples e foi amplamente utilizado no século XIX. As maiores críticas que este método recebe nos dias atuais é a de que alterações meteorológicas interferem no resultado apresentado; e a presença de espécies químicas oxidantes e redutoras provocam perturbações positivas e negativas, respectivamente, tornando a medição incerta (CALVERT et al., 2015; TIWARI; AGRAWAL, 2018). No entanto, este método continua possuindo uma vantagem notável: sua fácil aplicação, até mesmo por pessoas fora do ambiente científico-acadêmico.

Apesar de sua margem de erro ser significativamente maior do que as de técnicas mais modernas de monitoramento de ozônio, este método ainda é útil para estimar a concentração deste gás em áreas externas e internas, levando em consideração a umidade relativa medida em conjunto com a amostragem (ISAKSEN, 2012). O presente trabalho experimental objetivou utilizar o método de Schönbein para determinar as concentrações de ozônio em cinco pontos da cidade de Uberlândia (MG), em triplicata; comparar os resultados para definir os locais da cidade com concentrações mais significativas; construir um mapa demarcando os pontos cuja concentração de $\mathrm{O}_{3}$ foi aferida, com as concentrações representadas em escala; e realizar a extensão universitária deste conhecimento para a comunidade ao apresentar os resultados em uma escola pública de ensino médio para motivar o interesse científico dos alunos. 


\section{Área de estudo}

\section{MATERIAL E MÉTODOS}

O município de Uberlândia possui população estimada de 683.247 habitantes, se estabelecendo como o segundo município mais populoso do estado de Minas Gerais (IBGE, 2018). No entanto, poucos trabalhos foram publicados com estimativa da concentração de ozônio troposférico na cidade, não existindo, portanto, o monitoramento de variações temporais e espaciais de concentração deste poluente. Fica evidente a necessidade não apenas de realizar tais medições regulares, como também de alertar a comunidade sobre os perigos do ozônio à saúde e como fazer medições pontuais de maneira simples.

Para o presente trabalho, foram escolhidos cinco pontos da cidade para que fossem realizadas as medições, levando em consideração áreas de intenso fluxo veicular (P1 e P2), área de parque (P3), área afastada do centro urbano (P4) e área com alta atividade industrial em seu entorno (P5) (Quadro 1).

QUADRO 1. Identificação dos pontos de amostragem na cidade de Uberlândia-MG.

\begin{tabular}{|l|l|}
\hline Ponto $\mathbf{1}(\mathbf{P 1})$ & Campus "Santa Mônica" da UFU* \\
\hline Ponto $\mathbf{2}(\mathbf{P} 2)$ & Terminal Central \\
\hline Ponto $\mathbf{3}(\mathbf{P 3})$ & Parque do Sabiá \\
\hline Ponto $\mathbf{4}(\mathbf{P 4})$ & Campus "Glória" da UFU* \\
\hline Ponto 5 (P5) & Distrito Industrial \\
\hline \multirow{2}{*}{ * Universidade Federal de Uberlândia }
\end{tabular}

\section{Produção do amostrador}

O amostrador do método de Schönbein é constituído por uma tira de papel filtro embebida por uma mistura de amido de milho, iodeto de potássio $(\mathrm{KI})$ e água. A produção dessas tiras foi realizada em laboratório seguindo a metodologia da Flinn Scientific, Inc. (2016). Misturou-se $5 \mathrm{~g}$ de amido de milho em um béquer com $100 \mathrm{~mL}$ de água destilada, depois a solução foi aquecida até se tornar grossa e translúcida. Em seguida, $1 \mathrm{~g}$ de KI foi adicionada e a solução foi misturada até esfriar.

Com um pincel, espalhou-se a solução sobre os dois lados do papel filtro, posteriormente secando-o em forno a $50 \stackrel{\circ}{\circ} \mathrm{C}$ e recortando-o em tiras finas e uniformes. Um furo foi feito em cada tira para que a fixação do amostrador nos locais escolhidos usando barbante fosse possível e, finalmente, todas as tiras de papel foram guardadas em uma embalagem que as protegiam de correntes de ar e de luz solar direta. Ao utilizar o amostrador, este deveria ser novamente umedecido com água destilada, para reativá-lo, e pendurado livremente em áreas protegidas do vento e da radiação solar. Os compostos químicos presentes na tira de papel reagem com o ozônio atmosférico, após um período de exposição de oito horas, segundo as reações abaixo, colorindo o papel de roxo.

$$
\begin{gathered}
2 \mathrm{KI}+\mathrm{O}_{3}+\mathrm{H}_{2} \mathrm{O} \rightarrow 2 \mathrm{KOH}+\mathrm{O}_{2}+\mathrm{I}_{2} \\
\mathrm{I}_{2}+\text { amido } \rightarrow \text { complexo } \mathrm{I}_{2} \text {-amido (roxo) }
\end{gathered}
$$

\section{Análise dos resultados}

O resultado obtido com o amostrador é qualitativo e a relação entre a intensidade da coloração roxa obtida e a concentração de ozônio no local amostrado 
é diretamente proporcional. Para tornar este resultado semiquantitativo, foram necessários a escala cromática e o gráfico representados nas Figuras 1 e 2, respectivamente.

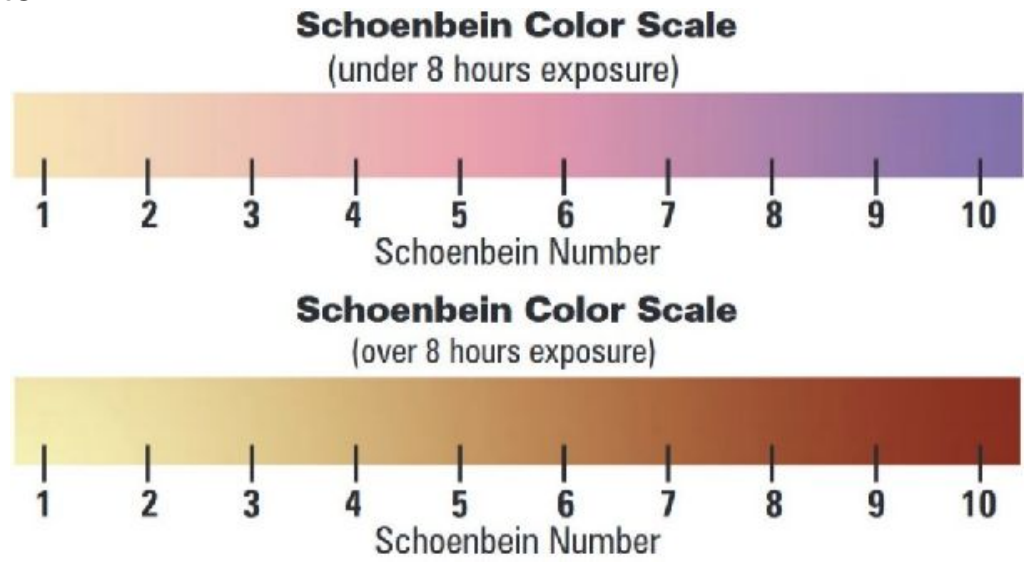

FIGURA 1. Relação entre a intensidade da cor e o Número de Schönbein. FONTE: IT'S OUR AIR (2014).

Nos momentos de instalação e de coleta dos amostradores, foi necessário realizar a medição da umidade relativa instantânea do local. O medidor utilizado foi o Lutron HT-3005. A média dos dois resultados foi cruzada com o Número de Schönbein obtido, fornecendo a concentração do ozônio troposférico em ppb.

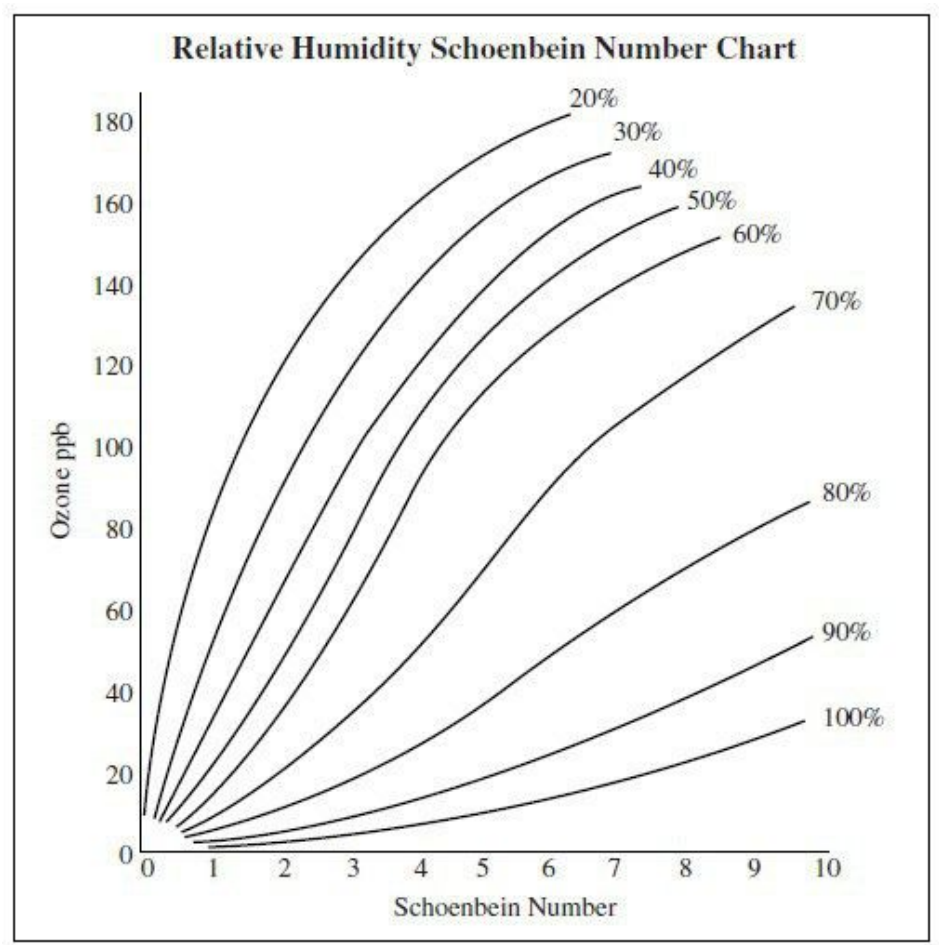

FIGURA 2. Relação entre o Número de Schönbein, a umidade relativa e a concentração de ozônio no local. FONTE: FLINN SCIENTIFIC (2016).

O tempo de amostragem escolhido foi das 8:00 às 16:00 horas, contemplando assim a necessidade de oito horas de exposição e o período diurno/vespertino, em que a concentração de ozônio é maior em comparação ao período noturno (ABDUL-WAHAB et al., 2005). A amostragem foi realizada no dia 23 de outubro de 2018 e não houve precipitação no dia. Além disso, em cada ponto 
de amostragem foram colocados três amostradores, espaçados por cerca de dez metros entre si. A Figura 3 exemplifica como foi feita a fixação dos amostradores nos locais escolhidos. Os cuidados com relação à interferência de ventos e luz solar foram tomados.

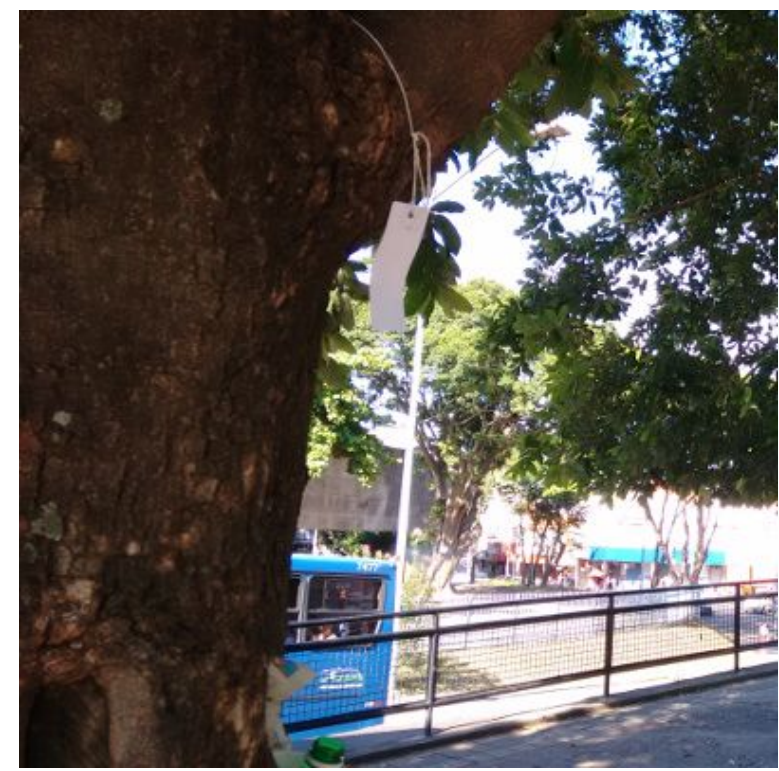

FIGURA 3. Amostrador fixado no ponto 2, Terminal Central, em Uberlândia-MG. FONTE: Autores, (2018).

\section{RESULTADOS E DISCUSSÃO}

Após o período de amostragem, os papéis foram novamente umedecidos com água destilada, para que a leitura fosse possível, e a cor obtida foi cruzada com a escala da Figura 1, determinando um Número de Schönbein (NS) para cada tira de papel. Este, por sua vez, foi cruzado com a média das umidades relativas (U\%) medidas durante a fixação e coleta dos papéis, segundo a Figura 2, determinando a concentração do ozônio troposférico $\left(\left[\mathrm{O}_{3}\right]\right)$, em ppb, para cada amostrador. Todos estes resultados foram organizados na Tabela 1.

TABELA 1. Resultados de NS, U\% e $\left[\mathrm{O}_{3}\right]$ para cada papel amostrador.

\begin{tabular}{|c|c|c|c|c|}
\hline Local & Ponto & U\% & NS & {$\left[\mathrm{O}_{3}\right]$} \\
\hline \multirow{3}{*}{$\begin{array}{l}\text { UFU Santa } \\
\text { Mônica }\end{array}$} & $\mathrm{P} 1 \mathrm{~A}$ & \multirow{3}{*}{$55,70 \%$} & 3 & $70 \mathrm{ppb}$ \\
\hline & P1B & & 4 & $105 \mathrm{ppb}$ \\
\hline & $\mathrm{P} 1 \mathrm{C}$ & & 4 & $105 \mathrm{ppb}$ \\
\hline \multirow{3}{*}{$\begin{array}{l}\text { Terminal } \\
\text { Central }\end{array}$} & P2A & \multirow{3}{*}{$61,70 \%$} & 3 & $65 \mathrm{ppb}$ \\
\hline & P2B & & 2 & $35 \mathrm{ppb}$ \\
\hline & $\mathrm{P} 2 \mathrm{C}$ & & 1 & $15 \mathrm{ppb}$ \\
\hline \multirow{3}{*}{$\begin{array}{l}\text { Parque do } \\
\text { Sabiá }\end{array}$} & P3A & \multirow{3}{*}{$59,80 \%$} & 4 & $95 \mathrm{ppb}$ \\
\hline & P3B & & 2 & $35 \mathrm{ppb}$ \\
\hline & P3C & & 2 & $35 \mathrm{ppb}$ \\
\hline \multirow{3}{*}{ UFU Glória } & P4A & \multirow{3}{*}{$59,20 \%$} & 2 & $35 \mathrm{ppb}$ \\
\hline & P4B & & 1 & $15 \mathrm{ppb}$ \\
\hline & P4C & & 2 & $35 \mathrm{ppb}$ \\
\hline \multirow{2}{*}{$\begin{array}{c}\text { Distrito } \\
\text { Industrial }\end{array}$} & P5A & \multirow{2}{*}{$67,30 \%$} & 5 & $85 \mathrm{ppb}$ \\
\hline & P5B & & 3 & $40 \mathrm{ppb}$ \\
\hline
\end{tabular}

As letras de $A$ a $C$ na coluna dos pontos indicam as três repetições do experimento em triplicata. Um dos amostradores do ponto 5, Distrito Industrial, foi perdido, de modo que a média final foi realizada apenas com dois resultados. A Tabela 2 expõe a média e o desvio padrão dos resultados de cada local de amostragem. 
TABELA 2. Concentração média de $\mathrm{O}_{3}$ para cada ponto amostrado.

\begin{tabular}{cc}
\hline Ponto & Concentração de $\mathbf{O}_{3}$ \\
\hline P1 & $93,3 \mathrm{ppb} \pm 20,2$ \\
P2 & $38,3 \mathrm{ppb} \pm 25,2$ \\
P3 & $55,0 \mathrm{ppb} \pm 34,6$ \\
P4 & $28,3 \mathrm{ppb} \pm 11,5$ \\
P5 & $62,5 \mathrm{ppb} \pm 31,8$ \\
\hline
\end{tabular}

A baixa precisão desta metodologia fica explícita no desvio padrão elevado que as medições apresentaram. Mesmo minimizando os efeitos da incidência solar e das correntes de ar, a variação entre as medições pode ser elevada devido a outras espécies químicas oxidantes e redutoras presentes no local, além dos erros associados à leitura da cor final do papel filtro (COOPER et al., 2014). Portanto, este não é um método recomendado para realizar o monitoramento regular das concentrações de ozônio. Todavia, os resultados obtidos possibilitam a comparação das concentrações identificadas nas regiões amostradas da cidade e, com ressalvas - trabalhos aplicando metodologias mais precisas devem ser feitos para comprovar isto -, a estimativa das localidades de Uberlândia cujos perigos relacionados às altas concentrações de ozônio são maiores.

De Souza et al. (2018) realizaram medições do ozônio troposférico presente em Campo Grande (MS), levando em consideração a variação temporal deste poluente no ar, e constataram que, além de as concentrações serem maiores em meses mais quentes e secos, como no verão, a variação diária do ozônio também é significativa, com concentração média mínima de $40 \mathrm{ppb}$ às sete horas da manhã e máxima de $97 \mathrm{ppb}$ ao meio-dia. Apesar de a metodologia de Schönbein não contemplar a variação temporal de $\mathrm{O}_{3}$, os resultados da Tabela 2 podem ser encarados como médias diárias, de modo que, ao meio-dia, as concentrações de ozônio seriam ainda mais elevadas do que as aferidas por este método.

As concentrações dos pontos 1,3 e 5 superaram o padrão regulamentado pela resolução CONAMA no 491/18, de 50 ppb. O ponto 1 (UFU Santa Mônica), inclusive, chegou próximo à marca dos 100 ppb, que a resolução classifica como "nível de atenção" e que pode causar problemas respiratórios tanto em crianças como em adultos (IT'S OUR AIR, 2014). Para melhor visualização da distribuição espacial das concentrações, o mapa da Figura 4 foi construído. Os círculos roxos possuem diâmetro proporcional à concentração de ozônio apresentada pelos pontos que representam. 


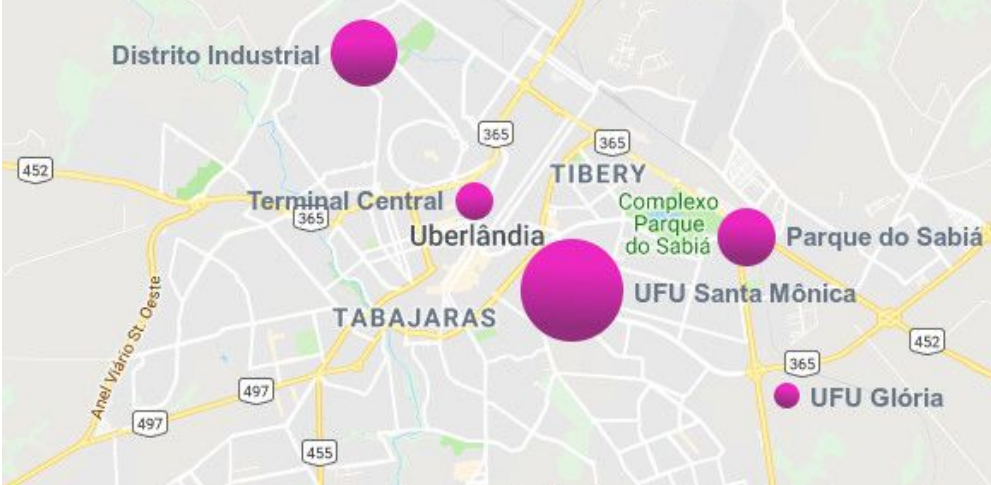

FIGURA 4. Mapa de distribuição espacial do ozônio em Uberlândia-MG.

FONTE: Autores, (2018).

Conforme o esperado, a maior concentração observada localiza-se no centro da cidade, em uma região com intenso fluxo de veículos automotores. No Terminal Central (P2), a concentração foi baixa, apesar do constante trânsito de ônibus. Uma possível explicação para este fenômeno é a de que existe uma distância entre o local de emissão dos precursores do $\mathrm{O}_{3}$ e o local de síntese do $\mathrm{O}_{3}$ a partir de seus precursores, principalmente por ação dos ventos.

Chang e Lee (2006) realizaram o monitoramento das concentrações de ozônio durante 10 anos, de 1994 a 2003, na cidade de Taipé, a capital de Taiwan. A frota veicular da cidade nos anos em que a pesquisa foi realizada era composta por 700 mil carros e mais de um milhão de motocicletas. Em todos os anos monitorados houveram dias excedendo o limite de $120 \mathrm{ppb}$, evidenciando a relação direta existente entre os poluentes emitidos por fontes veiculares e as altas concentrações de ozônio troposférico.

O ponto 3 (Parque do Sabiá) também apresentou concentrações elevadas, o que talvez se deva ao fato de que as amostragens foram feitas na extremidade do parque, próximo a uma avenida, e não no interior do mesmo. O Distrito Industrial apresentou a segunda maior concentração, devido à acentuada atividade industrial que ocorre no local. Já a menor das concentrações foi observada no campus Glória da UFU, também se alinhando com o que era esperado, uma vez que o campus se encontra afastado do centro urbano da cidade.

Francisco et al. (2016) realizaram medições da concentração de $\mathrm{O}_{3}$ na cidade de Araraquara (SP) utilizando amostradores passivos, como os do método de Schönbein, e amostradores ativos da CETESB (Companhia Ambiental do Estado de São Paulo). Comparando os resultados, contatou-se que os amostradores passivos apresentam resultados ligeiramente maiores, em relação aos amostradores ativos, mais precisos. Portanto, a concentração de ozônio em Uberlândia pode ser um pouco menor do que a apresentada na Tabela 2, mas não de modo significativo.

Os resultados do trabalho foram apresentados para os alunos do $1^{\circ}, 2^{\circ}$ e $3^{\circ}$ anos do ensino médio da Escola Estadual Professor Inácio Castilho, em Uberlândia, juntamente com as tiras de papel filtro utilizadas e a exposição da metodologia simples aplicada. A apresentação na escola teve o objetivo de mostrar aos alunos como funciona uma pesquisa científica, alertá- 
los para causas ambientais (educação ambiental) e motivá-los a utilizar a ciência como ferramenta para entender e tentar melhorar o planeta.

\section{CONCLUSÃO}

Os resultados encontrados neste trabalho ajudam a mostrar a concentração de ozônio média na cidade de Uberlândia e sua distribuição espacial. Três dos pontos analisados apresentaram concentrações maiores do que permite a legislação. Recomenda-se que estudos mais elaborados, que apliquem metodologias mais precisas e que contemplem a variação temporal deste poluente, sejam realizadas no futuro. Caso as concentrações elevadas dos pontos que superaram o padrão se mostrem normais, programas para controlar a emissão de poluentes primários precursores do ozônio devem ser implementados. Embora os resultados provenientes da metodologia de Schönbein se mostrem instáveis e não indicados para monitoramentos de longo prazo, este método se mantém funcional e apresenta, como maior vantagem, boa aplicabilidade para a educação ambiental em escolas.

\section{REFERÊNCIAS}

ABDUL-WAHAB, S. A.; BAKHEIT, C. S.; AL-ALAWI, S. M. Principal component and multiple regression analysis in modelling of ground-level ozone and factors affecting its concentrations. Environmental Modelling \& Software, v. 20, n. 10, p. 12631271, 2005. Disponível em: <https://doi.org/10.1016/j.envsoft.2004.09.001>. doi: 10.1016/j.envsoft.2004.09.001

BAIS, A. F.; MCKENZIE, R. L.; BERNHARD, G.; AUCAMP, P. J.; ILYAS, M. et al. Ozone depletion and climate change: impacts on UV radiation. Photochemical \& Photobiological Sciences, v. 14, n. 1, p. 19-52, 2015. Disponível em: <https://doi.org/10.1039/c4pp90032d>. doi: 10.1039/c4pp90032d

BRASIL. Resolução CONAMA no 491, de 19 de novembro de 2018. Publicado no D.O.U. Disponível em: <http://www2.mma.gov.br/port/conama/legiabre.cfm?codlegi=740>. Acesso em 17 mar. 2019.

CALVERT, J. G.; ORLANDO, J. J.; STOCKWELL, W. R.; WALLINGTON, T. J. The Mechanisms of Reactions Influencing Atmospheric Ozone. Oxford University Press, p. 16-18.2015.

CHANG, S. C.; LEE, C. T. Ozone variations through vehicle emissions reductions based on air quality monitoring data in Taipei City, Taiwan, from 1994 to 2003. Atmospheric Environment, v. 40, n. 19, p. 3513-3526, 2006. Disponível em: $<$ https://doi.org/10.1016/j.atmosenv.2006.01.038>. 10.1016/j.atmosenv.2006.01.038

COOPER, O. R.; PARRISH, D. D.; ZIEMKE, J.; CUPEIRO, M.; GALBALLY, I. E. et al. Global distribution and trends of tropospheric ozone: An observation-based review. 2014. Disponível em: <https://doi.org/10.12952/journal.elementa.000029>. doi: 10.12952/journal.elementa.000029 
DE SOUZA, A.; ARISTONE, F.; GARCIA, A. P.; DA SILVA SANTOS, D. A.; NÓBREGA, L. Estudo da associação entre óxidos de nitrogênio e concentração de ozônio com parâmetros meteorológicos. Geosul, v. 33, n. 68, p. 164-183, 2018. Disponível em: <https://doi.org/10.5007/2177-5230.2018v33n68p164>. doi: 10.5007/2177-5230.2018v33n68p164

FLINN SCIENTIFIC, Inc. Ozone Test Paper. 2016. Disponível em: $<$ https://www.flinnsci.com/api/library/Download/e057b1e3331d48099ae4ab4beeb5cb d4>. Acesso em: 17 mar. 2019.

FRANCISCO, A. P.; ALVIM, D. S.; GATTI, L. V.; PESQUERO, C. R.; ASSUNÇÃO, J. V. Ozônio troposférico e compostos orgânicos voláteis em região impactada pela agroindústria canavieira. Química Nova, v. 39, n. 10, p. 1177-1183, 2016. Disponível em: <https://doi.org/10.21577/0100-4042.20160132>. doi: 10.21577/0100-4042.20160132

HEGGLIN, M. I.; FAHEY, D. W.; MCFARLAND, M.; MONTZKA, S. A.; NASH, E. R. et al. Twenty Questions and Answers About the Ozone Layer: 2014 Update Scientific Assessment of Ozone Depletion: 2014. 2015. Disponível em: <http://wedocs.unep.org/bitstream/handle/20.500.11822/26719/775320QA_14Update .pdf? sequence=1\&isAllowed=y>. Acesso em: 17 mar. 2019.

IBGE - Instituto Brasileiro de Geografia e Estatística. Município de Uberlândia Panorama. 2018. Disponível em: <https://cidades.ibge.gov.br/brasil/mg/uberlandia/panorama>. Acesso em: 17 mar. 2019.

ISAKSEN, I. S. A. (Ed.). Tropospheric Ozone: Regional and Global Scale Interactions. Springer Science \& Business Media, p. 65-68.2012.

IT'S OUR AIR. Making and using ozone indicators. Module 1: Activity 6. NC Air Awareness $\quad$ Program. $2014 . \quad$ Disponível em: $<$ http://itsourair.org/sites/default/files/activities/IOA_1-6_Act_Ozone_Ind.pdf>. Acesso em: 17 mar. 2019.

MONKS, P. S.; ARCHIBALD, A. T.; COLETTE, A.; COOPER, O.; COYLE, M. et al. Tropospheric ozone and its precursors from the urban to the global scale from air quality to short-lived climate forcer. Atmospheric Chemistry and Physics, European Geosciences Union, v. 15, n. 15, p. 8889-8973, 2015. Disponível em: <https://doi.org/10.5194/acp-15-8889-2015>. doi: 10.5194/acp-15-8889-2015

SEINFELD, J. H.; PANDIS, S. N. Atmospheric Chemistry and Physics: From Air Pollution to Climate Change. John Wiley \& Sons, p. 44-47.2016.

TIWARI, S.; AGRAWAL, M. Tropospheric Ozone and Its Impacts on Crop Plants: A Threat to Future Global Food Security. Springer, p. 3-5.2018. 